\title{
Role of Magnetic Resonance Imaging in Evaluation of Neonatal and Pediatric Adnexal Masses \\ A.F.Yousof ${ }^{1}$, H.M.Khater ${ }^{1}$ and R.M.Khattab ${ }^{2}$ \\ ${ }^{1}$ Radio-diagnosis Dept., Faculty of Medicine, Benha Univ., Benha, Egypt \\ ${ }^{2}$ M.Sc., Zagazig Univ., Faculty of Medicine, Egypt \\ E-Mail:redakhattab1975@gmail.com
}

\begin{abstract}
Ovarian masses are most commonly observed in adults; they rarely occur in children. The majority of the ovarian masses encountered in children or patients of pre- menarchal age are non-neoplastic lesions. The clinical signs and symptoms of ovarian masses are usually non-specific. Early management may be necessary to preserve fertility. Gynecological malignant conditions constitute approximately $3 \%$ of all types of cancer in children. Ovarian tumors in children account for only $1 \%$ of childhood malignancies. However, the true incidence of malignant ovarian tumors in the pediatric population is unknown. is to study the role of magenetic resonace (MR) imaging in evaluation of neoplastic and non-neoplastic masses arising from uterine adnexa in neonates and pediatric age groups. During a period of 12 months (from December 2017 to December 2018), 100 consecutive patients (age range, 1-14 years; mean age, 8.8 years) with different clinical presentations were enrolled in this study. There was a highly significant reduction in the overall sexual satisfaction after hysterectomy $(\mathrm{p}<0.001)$. On the basis of the findings, the study concludes that MRI is good modality for differentiation of benign and malignant adnexal masses. Certain imaging features and the degree of enhancement on MRI images are helpful in differentiating adnexal masses. Thus, imaging findings may contribute incremental value to clinical parameters in providing prognostic information, consequently improving the quality of the data used in therapeutic planning.
\end{abstract}

Keywords : Adnexal Masses , Magnetic Resonance Imaging, Neonatal ovarian masses.

\section{Introduction}

The most common adnexal abnormality in the pediatric population is an ovarian cyst. Ovarian cysts are the most frequent cause of an abdominal mass in the fetus and in the new born. An anechoic focus in an ovary is considered a follicle if it is smaller than 3.0 $\mathrm{cm}$. A mature dominant follicle may fail to involute. Appropriately and may enlarge into a functional cyst or corpus luteum. Rupture or hemorrhage often brings these patients to medical attention. Based on the degree of complexity associated with blood products, clot formation, lysis, and retraction, appearances variable on all cross-sectional imaging modalities. Whether simple or complex, these lesions are followed with ultrasound to confirm resolution and exclude cystic neoplasm [10].

Ovarian neoplasms commonly have a cystic component and may be benign or malignant. The solid component is the most statistically significant predictor of malignancy. Categories are defined by the cell of origin: germ cell tumors, epithelial tumors, and stromal tumors. These neoplasms generally present in girls as a result of pain, increasing abdominal girth, and symtoms derived from hormonal effects when masses are functional. The presence of fat or calcification in the lesion is diagnostic and easily distinguished by MRI [1].

The accurate diagnosis of ovarian torsion is imperative, as loss of the ovary can have long-term consequences in terms of fertility. However, a nonspecific clinical presentation in conjunction with a highly variable imaging appearance makes the diagnosis of ovarian torsion challenging. This is complicated even further in the pediatric population, as these patients cannot always articulate their symptoms or provide an adequate medical history. Therefore, imaging has a critical role in the diagnosis of ovarian torsion in pediatric patients. A coexistent mass within the ovary may or may not be present. If advanced imaging is required, MRI without and with cantrast materail can confirm the diagnosis and may highlight any underlying mass [10].

Pelvic infection may emanate from the adnexa or may involve the adnexal regions from adjacent structures, creating an imaging appearance similar to that of neoplasms with complicating features or torsion. Classic imaging findings of tubo-ovarian abscess and pyosalpinx are complex fluid collections with thick walls and rim enhancement or increased peripheral vascular flow, often with adjacent inflammatory change and free fluid. Ultrasound depicts septations well, but MRI offers a global assessment, which is often required preoperatively [2].

\section{Subjects and methods}

During a period of 12 months (from December 2017 to December 2018), 100 consecutive patients (age range, 1-14 years; mean age, 8.8 years) with different clinical presentations were enrolled in this study.

They were referred from surgical department presented with adnexal masses by ultrasound examination who underwent preoperative MRI in department of radiology Zagazig university hospital, Egypt. Patients were passed to operative exploration for histopathological diagnosis. The median time from scanning to surgery was 35 days from initial ultrasound scanning (with MRI) to surgery. Out of 102 cases enrolled in this study, two patients were excluded from the study because their parents refused surgical 
exploration. Only 100 patients were underwent surgical exploration throughout the study.

The following imaging features were used for diagnosis of malignant lesions on MRI: irregularity, vegetation on the wall, solid-cystic lesion, large size of the lesion presence septum in cystic lesion and early contrast enhancement (Gadolinium) the presence of ascites or adenopathy were the factors more indicative of malignancy, while presence of fat, hemorrhage, no septum in cystic lesions and no contrast enhancement were the feature of benignancy.

Patients that fulfill the inclusion criteria will be subjected to: Full detailed history, Clinical examination, Laboratory work-up, Ultrasound and MRI.

\section{Results}

Table (1) This table shows that patients age range from 1 to 14 year with mean age of $(8.8 \pm 0.74)$.

Fig (1) In this table, $40 \%$ of patients presented with pain, $19 \%$ with fever, $20 \%$ with palpable mass and $9 \%$ with urinary symptoms.

Table (2) Out of lesions (12\%) were found in age group 1-2 years, $(26 \%)$ in 3-5 years, $(24 \%)$ in $6-8$ years, $(15 \%)$ in $9-11$ years and $(23 \%)$ were found in age group 12-14 years.

Fig (2) The most common site of the adnexal masses in both malignant and benign were on the right site of the ovaries $(62 \%)$.

Table (3) In this table, solid-cystic lesion was the high percentage among the malignancy features seen (80\%), followed by early contrast enhancement (40\%), while the least percentage among the malignancy features was seen in solid only feature $(6.7 \%)$

Fig (3) Results of comparison between MRI imaging and histopathology found that 95 of the 100 adnexal masses were identified as tumor lesions by MRI; of which 70 were benign lesions and 30 were malignant lesion. Also the sensitivity of MRI for detection of malignant lesions was $93.3 \%$. Specificity: 95.7\%, Positive predictive value: $90.3 \%$ and Negative predictive value: $97.1 \%$. Results also found that the accuracy of MRI was $95 \%$.

Table (1) Demographic characteristics of patients.

\begin{tabular}{lc}
\hline Demographic data & $\begin{array}{c}\text { All studied } \\
\text { patients }(\mathbf{N}=\mathbf{1 0 0})\end{array}$ \\
\hline Age (years) & \\
Mean \pm SD & $8.8 \pm 0.74$ \\
Median (Range) & $11(1-14)$ \\
\hline
\end{tabular}

Table (2) Distribution of adnexal masses in relation to age of patients.

\begin{tabular}{ll}
\hline Age (Years) & No. $(\%)$ \\
\hline $1-2$ & $12(12 \%)$ \\
$3-5$ & $26(26 \%)$ \\
$6-8$ & $24(24 \%)$ \\
$9-11$ & $15(15 \%)$ \\
\hline
\end{tabular}

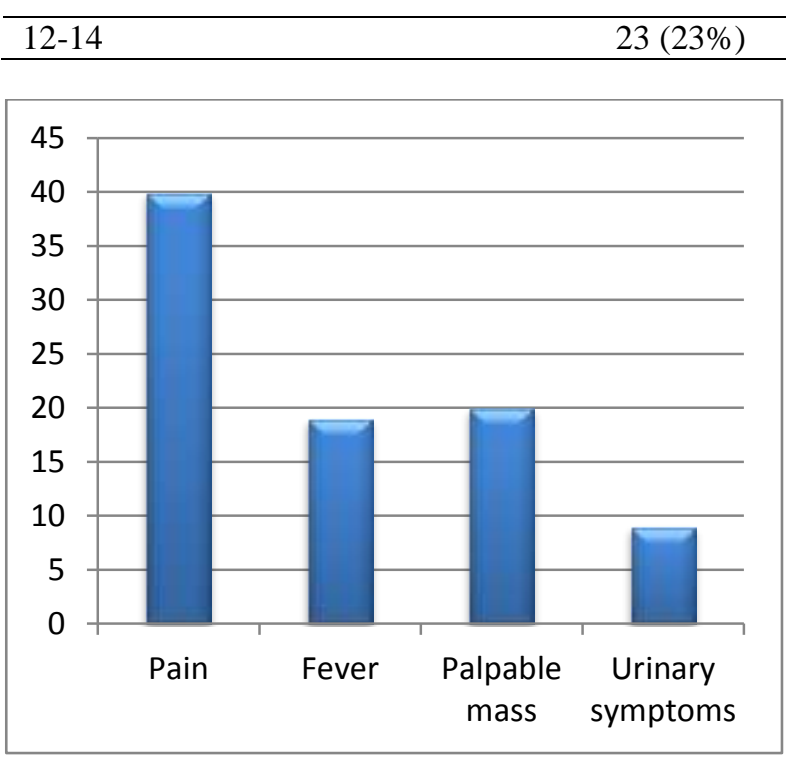

Fig (1) Distribution of clinical presentation.

Table (3) Distribution of adnexal mass in relation to site of ovaries.

\begin{tabular}{ll}
\hline Site & No. (\%) \\
\hline Right & $62(62 \%)$ \\
Left & $38(38 \%)$ \\
\hline
\end{tabular}

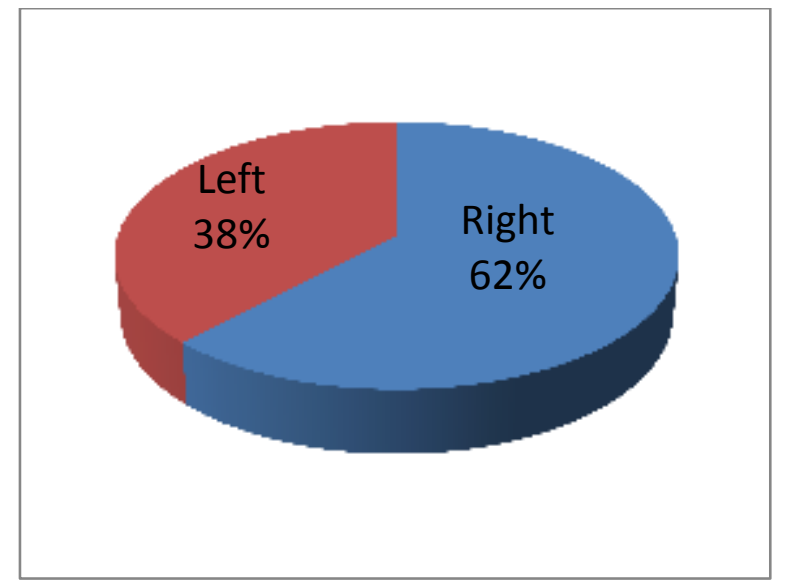

Fig (2) Distribution of adnexal mass in relation to site of ovaries.

Table (6) Characteristic Features of 30 malignant adnexal masses on MRI imaging presence.

\begin{tabular}{lc}
\hline Feature & No. (\%) \\
\hline Diameter greater than $\mathbf{6} \mathbf{~ c m}$ & $12 / 30(40 \%)$ \\
Solid only & $2 / 30(6.7 \%)$ \\
Solid-cystic & $24 / 30(80 \%)$ \\
\hline Cystic only & $4 / 30(13.3 \%)$ \\
Septum & $9 / 30(30 \%)$ \\
Vegetation on the wall & $6 / 30(20 \%)$ \\
Early enhancement & $12 / 30(40 \%)$ \\
Peritoneal invasion & $8 / 30(26.7 \%)$ \\
Lymphadenopathy & $7 / 30(23.3 \%)$ \\
\hline
\end{tabular}




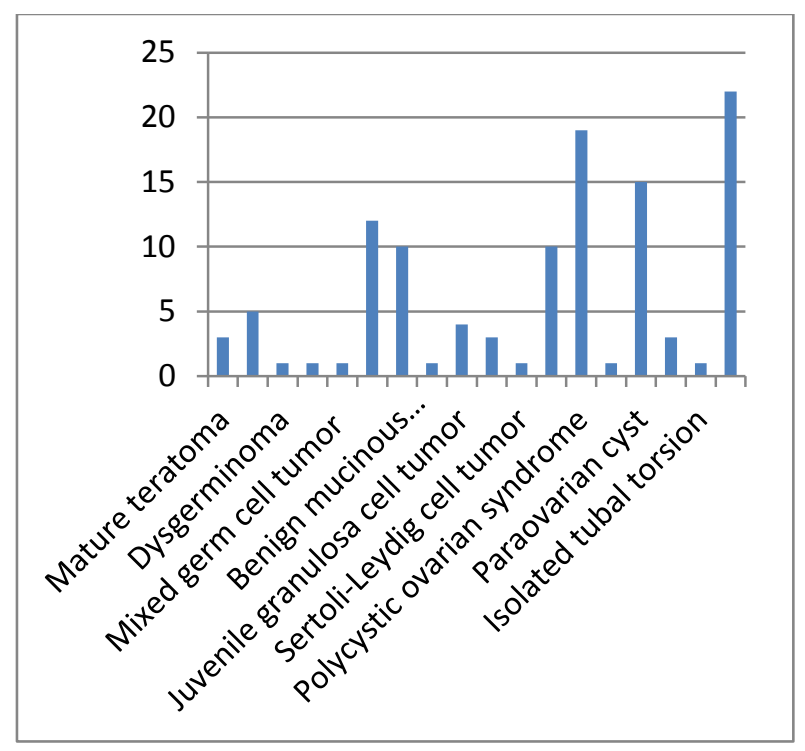

Fig (3) Distribution of cases according to final Diagnosis.

\section{Case presentations}

Case (1) Mature cystic teratoma in a 16-year-old girl.

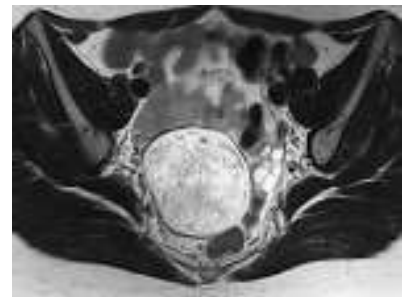

a)

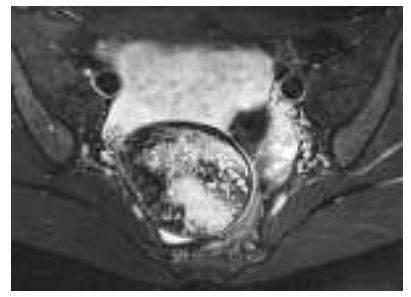

b)
Fig (4) a) Coronal oblique T2-weighted image through the pelvis shows heterogeneous predominantly $\mathrm{T} 2$ hyperintense mass. b) Axial fat-suppressed T2weighted image shows loss of signal within the peripheral and central portions of lesion, consistent with fat.

Case (2) Mucinous cystadenoma in an 11-year-old girl.

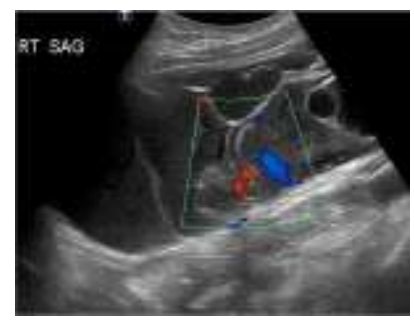

a)

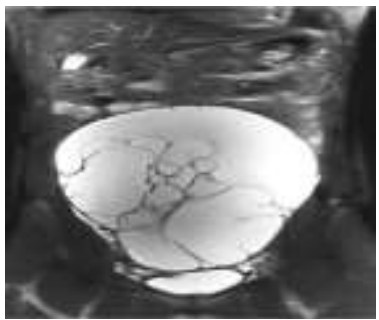

b)
Fig. (5) a,b) Sagittal Doppler ultrasound image (a) and coronal single-shot T2-W fat saturated MR image (b) demonstrate a large multicystic mass composed of multiple variable-size cysts with vascularity in some of the septa (arrow). Pathology revealed mucinous cystadenoma.

\section{Discussion}

Malignant lesions typically require oophorectomy and a potentially different surgical approach. Because the optimal approach for benign and malignant adnexal lesions differs substantially, preoperative diagnosis and risk stratification is a critical component of gynecological imaging.

So this study aimed to describe the role of magenetic resonace (MR) imaging in evaluation of neoplastic and non-neoplastic masses arising from uterine adnexa in neonates and pediatric age groups.

Our patients had age ranged from 1 to 14 year with mean age of (8.8 \pm 0.74$)$. Their presentations were $40 \%$ presented with pain, $19 \%$ with fever, $20 \%$ with palpable mass and $9 \%$ with urinary symptoms.

Out of lesions (12\%) were found in age group 1-2 years, $(26 \%)$ in $3-5$ years, $(24 \%)$ in $6-8$ years, $(15 \%)$ in 9-11 years and $(23 \%)$ were found in age group 1214 years.

In our study, the most common site of the adnexal masses in both malignant and benign were on the right site of the ovaries $(62 \%)$.

Ovarian neoplasms that occur in children are the same as in adults but differ in frequency. They can be classified into four types: germ cell tumors, 60-80\%; surface epithelial-stromal tumors, 15-20\%; sex cordstromal tumors, 10-20\%; and miscellaneous tumors (gonadoblastoma, lymphoma/ leukemia, small cell carcinoma, soft-tissue tumors), $<5 \%$ (5). In contrast, epithelial tumors are the most common ovarian neoplasm in adults. Other adnexal masses include hemorrhagic ovarian cyst, massive ovarian edema, and tubo-ovarian lesions such as tubo-ovarian torsion, tubo-ovarian abscess (TOA), hydrosalpinx and paraovarian cysts. In addition, uterine, bowel and appendiceal masses can mimic an adnexal mass on presentation.

In the current study, the ovarian germ cell tumors represent $(10 \%)$ of adnexal mass, ovarian surface epithelial-stromal tumors represent $(12 \%)$ of adnexal mass, ovarian sex cord-stromal tumors represent $(8 \%)$ of adnexal mass, ovarian non-neoplastic processes represent $(30 \%)$ of adnexal mass and tubo-ovarian masses represent (40\%) of adnexal mass.

In the current study, the ovarian germ cell tumors represent $(10 \%)$ of adnexal mass, which included mature teratoma $(5 \%)$, immature (malignant) teratoma $(3 \%)$, dysgerminoma (1\%), yolk sac tumor $(1 \%)$ and mixed germ cell tumor $(1 \%)$.

Studies showed that the epithelial ovarian tumors represent $15-20 \%$ of all pediatric ovarian tumors [3] but occur almost exclusively post-menarche and hormonal stimulation has been proposed to trigger their development [8]. Histological subtypes of epithelial ovarian tumors include serous, mucinous, endometrioid, clear cell, transitional (Brenner) and epithelial-stromal types. The vast majority of ovarian epithelial tumors in the pediatric population are serous or mucinous type [11]. Each subtype can be graded as benign, borderline or malignant. Borderline or 
malignant epithelial tumors occur in only $2-3 \%$ of women younger than 21 years with an ovarian neoplasm but represent a much higher proportion of cases at tertiary referral centers, ranging from $15 \%$ to $35 \%$ [12].

Similar to our study results which found that ovarian surface epithelial-stromal tumors represent (12\%) of adnexal mass of which benign serous cystadenoma $(10 \%)$, benign mucinous cystadenoma (1\%) and borderline or malignant epithelial tumor $(1 \%)$.

In our study ovarian sex cord-stromal tumors represent $(8 \%)$ of adnexal mass of which juvenile granulosa cell tumor (4\%), thecoma-fibroma tumor (3\%), and sertoli-leydig cell tumor $(1 \%)$.

This is in agreement with another studies which reported that Ovarian sex cord-stromal tumors represent 10-20\% of pediatric ovarian neoplasms [7]. Ovarian sex cord-stromal tumors often have more than one cell type within the tumor. As a group, ovarian sex cord-stromal tumors occur across all age groups, but the distribution of histological types among ages is vastly different. Granulosa cell and Sertoli-Leydig cell tumors are the most common in children and adolescents while thecoma-fibromas are rare [4] the opposite distribution is the case for women. Most sex cord-stromal tumors are benign or of low grade at presentation [4].

Also our study results found that ovarian nonneoplastic processes represent $(30 \%)$ of adnexal mass of which hemorrhagic cyst $(10 \%)$, polycystic ovarian syndrome (19\%) and massive ovarian edema (1\%).

Our study results found that tubo-ovarian masses represent $(40 \%)$ of adnexal mass of which paraovarian cyst $(14 \%)$, tubo-ovarian torsion $(3 \%)$, isolated tubal torsion (1\%) and tubo-ovarian abscess (22\%).

Similar to other studies in which tubo-ovarian torsion accounts for $30 \%$ of pediatric ovarian surgery and occurs in a bimodal distribution, with $16 \%$ of cases occurring in infants younger than 1 year and $52 \%$ occurring in children ages 9-14 years. An associated lead point is present $50 \%$ of the time, which is a malignant neoplasm in $2 \%$.

In the current study, solid-cystic lesion was the high percentage among the malignancy features seen $(80 \%)$, followed by early contrast enhancement (40\%), while the least percentage among the malignancy features was seen in solid only feature (6.7\%).

Study also found that malignant adnexal mass has strong and early enhancement than benign adnexal mass. This pattern of greater enhancement is similar to the observations made in studying tumors in other parts of body [9]. MRI imaging is highly accurate in the diagnosis of lesion in adnexal and the best predictors of malignancy are solid in cystic lesion and early contrast enhancement.

\section{Conclusion}

On the basis of the findings, the study concludes that MRI is good modality for differentiation of benign and malignant adnexal masses. Certain imaging features and the degree of enhancement on MRI images are helpful in differentiating adnexal masses. Thus, imaging findings may contribute incremental value to clinical parameters in providing prognostic information, consequently improving the quality of the data used in therapeutic planning.

\section{References}

[1] E.Y.Anthony, M.P.Caserta, J.Singh, M.Y.Chen, Ad $\neg$ nexal masses in female pediatric patients. AJR Am J Roentgenol, Vol.198(5), PP.426-431, 2012.

[2] K.Darge, S.A.Anupindi, D.Jaramillo, MR imaging of the abdomen and pelvis in infants, children, and adolescents. Radiology, Vol.261(1), PP.12-29, 2011.

[3] R.Forstner,I.Thomassin-Naggara, T.M.Cunha , ESUR recommendations for MR imaging of the songographically indeterminate adnexal mass: an update. Eur Radiol. Vol.27, PP.2248-2257, 2017.

[4] K.Hassen,M.A.Ghoussain P.Rousset , Characterization of papillary projections in benign versus borderline and malignant ovarian masses on conventional and color Doppler ultrasound. AJR Am J Roentgenol, Vol.196, PP.1444-1449, 2011.

[5] S.H.Heo, J.W.Kim,S.S.Shin , Review of ovarian tumors in children and adolescents: radiologicpathologic correlation. Radiographics, Vol.3, PP. 2039-2055, 2014.

[6] H.J.Kim, S.Y.Lee, Y.R.Shin, The value of diffusion-weighted imaging in the differential diagnosis of ovarian lesions: a meta-analysis. PLoS One, Vol.11, PP. 65-74, 2016.

[7] F.H.Ma, S.H.Zhao, J.W.Qiang, MRI appearances of mucinous borderline ovarian tumors: pathological correlation. J Magn Reson Imaging, Vol. 40, PP.745 -751, 2014.

[8] X.F.Meng, S.C.Zhu, S.J.Sun , Diffusion weighted imaging for the differential diagnosis of benign vs. malignant ovarian neoplasms. Oncol Lett, Vol.11, PP.3795-3802, 2016.

[9] A.R.Padhani and J.E.Husband, Dynamic contrast enhanced MRI studies using ECF agents, Clin Radiol; Vol. 56, PP.607-620,2001.

[10] A.Sintim-Damoa, A.S.Majmudar, H.L1.Cohen, L.S.Parvey ,Pediatric Ovarian Torsion: Spectrum of Imaging Findings. Radiographics, Vol.37 (6), PP.1892-1908,2017.

[11] X.Yuan, L.Guo, W.Du , Diagnostic accuracy of DWI in patients with ovarian cancer: a metaanalysis. Medicine, Vol.96, PP.66-59, 2017.

[12] S.H.Zhao, J.W.Qiang, G.F.Zhang , MRI in differentiating ovarian borderline from benign mucinous cystadenoma: pathological correlation. J Magn Reson Imaging, Vol.39, PP.162-166, 2014. 